\title{
Cellular Neural Networks with Effect from Friend of a Friend
}

\author{
Yoshihiro Kato $^{\dagger}$, Yoko Uwate ${ }^{\dagger}$ and Yoshifumi Nishio ${ }^{\dagger}$ \\ $\dagger$ Department of Electrical and Electronic Engineering, Tokushima University \\ 2-1 Minami-Josanjima, Tokushima, 770-8506 Japan \\ Email: \{kkato, uwate, nishio\}@ee.tokushima-u.ac.jp
}

\begin{abstract}
Generally, in the conventional CNN, each cell is connected to only its neighboring cells according to a template. In this case, the information that a cell can obtain from its neighboring cells is limited. In actual association, we tend to have a relation with another group that is the best friend belongs. Therefore, in this study, we propose cellular neural networks with effect from friend of a friend which took in the actual association to CNN.
\end{abstract}

\section{Introduction}

In recent years, our life teems with information by growth of high information society. Generally, digital circuit is used for many information processing. However, digital circuit cannot perform real time processing. Then in 1988, Cellular Neural Networks (CNN) [1] were introduced. The idea of the CNN was inspired from the architecture of the cellular automata and the neural networks. A different point from the conventional neural networks is that CNN has local connectivity property. The local connectivity property makes the CNN tailor made for VLSI implementation. Therefore, the CNN has the features of time continuity, spatial discreteness, nonlinearity and parallel processing capability. Furthermore, the structure of $\mathrm{CNN}$ resembles that of animals' retina. Therefore, CNN can be used for various image processing applications [2][6]. In CNN, each cell is connected to its neighboring cells according to a template. Then, each cell is influenced by neighboring cells and its value is updated.

As the proverb "Birds of a feather flock together" says, we are likely to become friendly with someone who have similar interests, values, purpose and so on. Hence, people with common principles and purpose organize a group. The larger scale of the group, the group has strong power. Therefore, large scale group is insusceptible to external influence. In job hunting, we join a company in empathy with corporate identity, business lineup and so on. And we are working as an employee. Although each employee has individuality at the beginning of company, they are taking on a company style through employee some training programs and works. In this way, on the society in which we live, the member has a color of the group, though each one has individuality. Furthermore, large companies have strength compared with small and medium-sized enterprises.

In this study, we propose the new method in consideration of the phenomena in such actual society, and investi- gate the effect in image processing. Generally, in the conventional $\mathrm{CNN}$, each cell is connected to only its neighboring cells according to a template. In this case, the information that a cell can obtain from its neighboring cells is limited. In the proposed method, combinations of among cells are decided by the value of each cell. In each cell, a cell with the nearest value of center cell is defined as "Best friend cell" in eight neighboring cells. Then, the state value of each cell is updated receive influences from the best friend cell and its neighboring cells. We show some simulation results and confirm its effectiveness. Especially, we confirm that the proposed method is effective as edgepreserving smoothing.

\section{Cellular Neural Networks}

In this section, we explain the conventional structure of the CNN. The CNN has $M$ by $N$ processing unit circuits called cells. The cell contains linear and nonlinear circuit elements which are typically linear capacitors, linear resistors, linear and nonlinear controlled sources. Cells are arranged in a reticular pattern to $M$ line $N$ row. We represent a cell $C(i, j)$ using a variable $i$ which denotes vertical position and a variable $j$ which denotes horizontal position. The CNN is an array of cells. Each cell is connected to its neighboring cells according to a template. Usually, the template is the same for all cells except for boundary cells. When the CNN is used for image processing, each cell of $\mathrm{CNN}$ is corresponded to each pixel of image.

State equation :

$$
\begin{aligned}
\frac{d v_{x i j}}{d t}= & -v_{x i j}+\sum_{k=i-r}^{i+r} \sum_{l=j-r}^{j+r} A_{(i, j ; k, l)} v_{y k l}(t) \\
& +\sum_{k=i-r}^{i+r} \sum_{l=j-r}^{j+r} B_{(i, j ; k, l)} v_{u k l}(t)+I,
\end{aligned}
$$

Output equation :

$$
v_{y i j}(t)=\frac{1}{2}\left(\left|v_{x i j}(t)+1\right|-\left|v_{x i j}(t)-1\right|\right),
$$

where $v_{x}, v_{y}$ and $v_{u}$ represent a state, an output and an input of cell, respectively. In the Eq. (1), $A$ is the feedback template and $B$ is the control template. These and the constant bias $I$ are collectively called general template. The output equation is a piece-wise linear function. In fact, the output value of CNN is within of -1 to 1 . When the CNN 
is used for image processing, values of black and white are treated as 1 and -1 , respectively.

\section{Cellular Neural Networks with Effect from Friend of a Friend}

In this section, we explain the algorithm of the cellular neural networks with effect from friend of a friend (FF$\mathrm{CNN}$ ). The FF-CNN is that the method of constituting a new combination. The algorithm is described as follows.

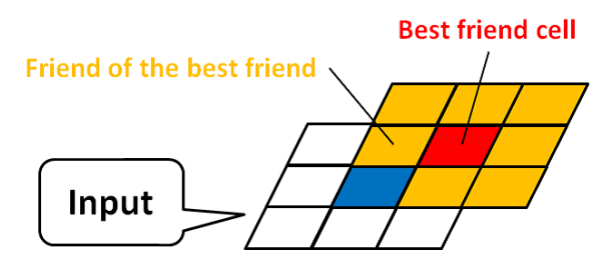

Figure 1: Definition the best friend cell in input image.

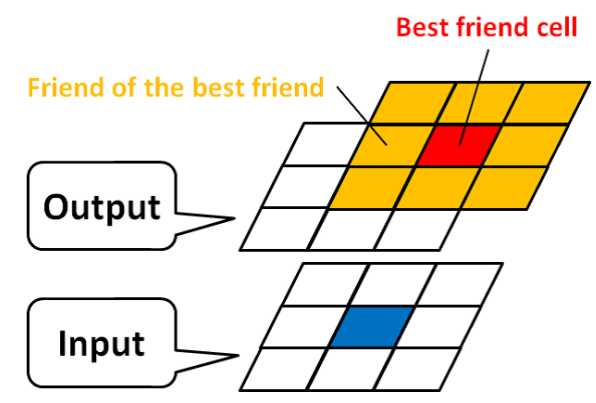

Figure 2: Definition the best friend cell in output image.

Step 1: In each cell of input image, a cell with the nearest value of center cell is defined as "Best friend cell" in eight neighboring cells.

Step 2: The combination centering on a cell $C(i, j)$ is changed into a combination centering on the best friend cell.

Moreover, the element of template of the best friend and the center cell are replaced. With this, a new combination is constituted like Fig. 1.

Step 3: The value of each cell is updated according to the Eqs. (1) and (2) using the new combination.

Step 4: In each cell of output image, a cell with the nearest value of center cell $C(i, j)$ in input image is defined as "Best friend cell" in eight neighboring cells like Fig. 2.

Step 5: Steps 2 to 4 are repeated every $0.0005[\tau]$.

In the proposed method, each cell in input image is used for basis of definition the best friend. Therefore, the state value of each cell is updated by receiving influences from the best friend cell and its neighboring cells.

\section{Simulation Results}

In this section, we show some simulation results for image denoising, edge-preserving smoothing and divide domains by using the FF-CNN. The used templates are found in [7]. In this study, image size is $128 \times 128$ pixels.

\subsection{Image Denoising}

In this subsection, we show some simulation results of image denoising by using the FF-CNN. Generally, in the conventional CNN, "S mall object remover" template [7] is used for image denoising. However, this template can not remove a big noise and output image is converged on black and white. In gray scale image, the conventional CNN can not perform with maintained gray scale. As the other way for image denoising, noisy image is defused.

In the FF-CNN, we use "Heat diffusion" template [7] for image denoising. Boundary condition is fixed to +1 .

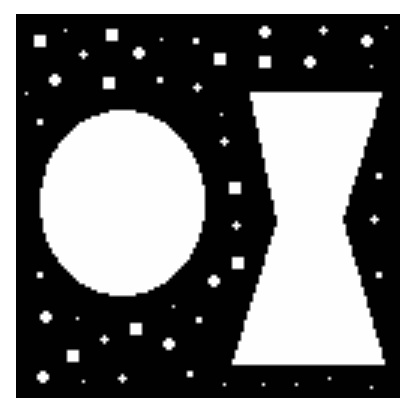

(a)

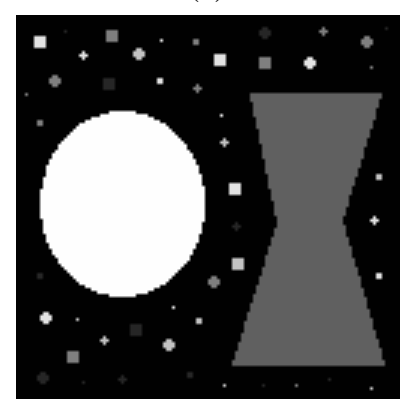

(c)

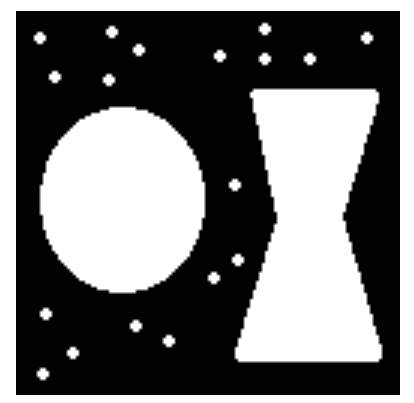

(b)

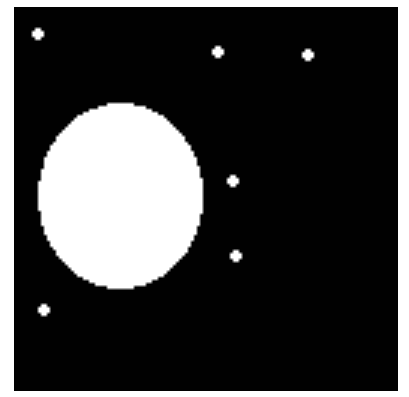

(d)
Figure 3: Image denoising by the conventional CNN using "S mall ob ject remover" template. (a) Binary input and initial state images. (b) Image denoising result for binary image. (c) Gray scale input and initial state images. (d) Image denoising result for gray scale image.

Figure 3(b) shows the image denoising result by the conventional CNN using "S mall object remover" template to binary image like Fig. 3(a). The noise of the small size is removed. However, the noise over certain size is not removed. Moreover, the angle of the object is waned.

Figure 3(d) shows the image denoising result by the conventional CNN using "S mall object remover" template to gray scale image like Fig. 3(c). Similar to the above re- 


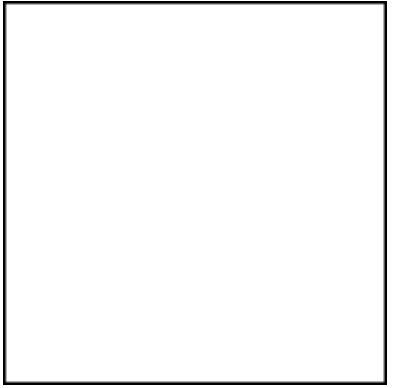

(a)

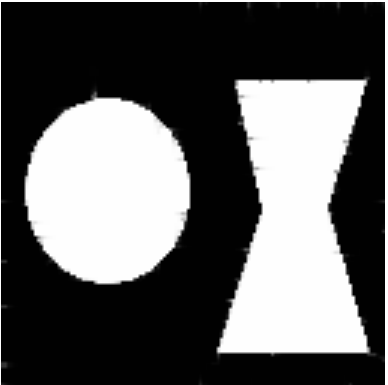

(b)

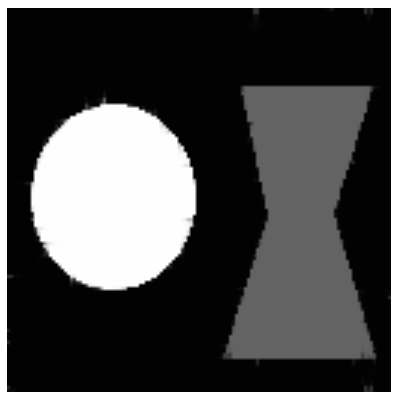

(c)

Figure 4: Image denoising by the FF-CNN. (a) Initial state image. (b) Image denoising result for binary image. (c) Image denoising result for gray scale.

sult, the noise over certain size is not removed. Moreover, an output image is converged on black and white when the "S mall object remover" template is used. Then, the right side object of the value near black has disappeared.

On the other hand, Fig. 4 shows the image denoising result by the FF-CNN using "Heat diffusion" template. Figure 4(b) shows the output image by using the FF-CNN to binary input image and the initial state image like Fig. 3(a) and Fig. 4(a). All noise in the input image were removed. Figure 4(c) shows the output image by using the FF-CNN to gray scale input image and the initial state image like Figs. 3(c) and Fig. 4(a). Similar to the above result, all noise in the input image were removed regardless of gray scale. Moreover, there is remained with keep the value of gray scale which is the object of the right side disappeared by the conventional CNN.

Additionally, we evaluate the performance of image denoising using the FF-CNN. The error value between the ideal output and the obtained output is calculated by

$$
\text { Error }=\sum_{i, j=0}^{127 \times 127}\left|v_{m i j}-v_{y i j}\right|
$$

where $v_{m}$ is the value of each cell in the ideal output.

Table 1 shows the error evaluation of image denoising using the conventional CNN and the FF-CNN. In every case, we confirm that the FF-CNN can remove some noise effectively than the conventional CNN. Especially, for the
Table 1: Error Evaluation

\begin{tabular}{|c|c|c|}
\hline & Conventional CNN & FF-CNN \\
\hline \hline Figure 3(a) & 400.844 & 101.078 \\
\hline Figure 3(c) & 2674.344 & 221.813 \\
\hline
\end{tabular}

gray scale image, the error is greatly reduced since the gray object remain in the output image.

From these results, we consider that the FF-CNN is more effective than the conventional CNN for image denoising.

\subsection{Edge-preserving Smoothing}

In this subsection, we show some simulation results of edge-preserving smoothing by the FF-CNN. In this simulation, we confirm that the phenomenon of "Each employee has individuality, however they are taking on a company style" as discussed earlier in introduction. The same "Heat dif fusion" template and boundary condition as subsection 4.1 are used. And Fig. 4(a) are used as initial state image.

Figure 3(c) has white, gray objects, and a black domain of a background. Dispersion is calculated on the basis of the value of each domain within the limits of $\pm(1,5,10$ and 20) [\%]. And the Fig. 3(c) which gave dispersion to each domain are used as input images.

Figure 5 shows the simulation results of edge-preserving smoothing by the FF-CNN. For the input image with the dispersion in several percent, we confirm that image denoising and smoothing can be performed effectively like Figs. 5(a) and (b). However, in near a boundary, disorder of a value occurs when the range of dispersion becomes large like Figs. 5(c) and (d).

From these results, we consider that the FF-CNN is effective as edge-preserving smoothing.

\subsection{Divide Domains}

In this subsection, we show other application using the FF-CNN. Since the cells of the similar value generated the domain, we thought that the FF-CNN was effective as application which divides sparse domain from thick domain in image. In this simulation, the choice of the best friend and the construction of the new combination which is only one time depending on an input value. To divide sparse domain from thick domain, we use "S moothing" template [7]. Boundary condition is using mirror reflector condition.

Figure 6(a) shows the output image by the conventional CNN using "S moothing" template. The thick domain of the left half in the input image which remains as a black domain. However, the sparse domain of the right half in the input image which is still a spot pattern. On the other hand, Fig 7(a) shows the output image by the FF-CNN using "S moothing" template. The thick domain of the left half in the input image remains as a black domain. At the same time, the sparse domain of the right half in the input 


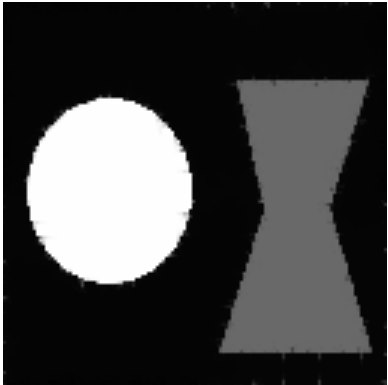

(a)

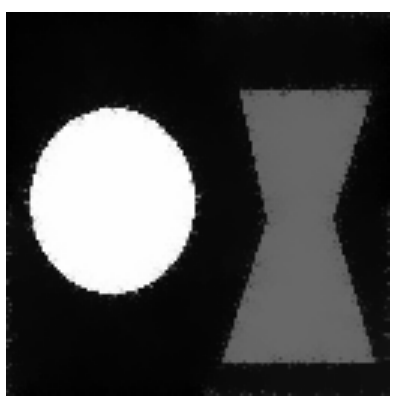

(c)

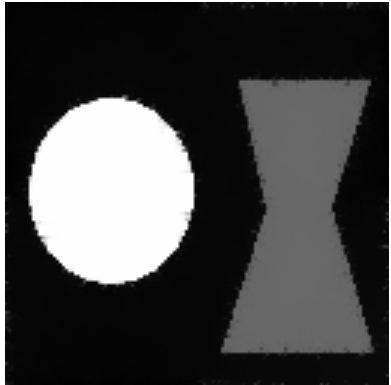

(b)

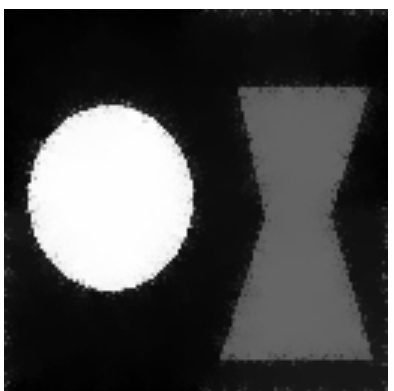

(d)
Figure 5: Edge-preserving smoothing by the FF-CNN. (a) The simulation result for the input image with disproportionate rate of \pm 1 [\%]. (b) The simulation result for the input image with disproportionate rate of $\pm 5[\%]$. (c) The simulation result for the input image with disproportionate rate of $\pm 10[\%]$. (d) The simulation result for the input image with disproportionate rate of $\pm 20[\%]$.

image remains as a white domain. Thereby, we can see that the thick domain and the sparse domain can be divided with the FF-CNN.

\section{Conclusions}

In this study, we have proposed cellular neural networks with effect from friend of a friend. The FF-CNN is that the new method in consideration of the phenomena in such actual society which likes "Birds of a feather flock together". From simulation results of image denoising, the noise of the size which was not removed by the conventional CNN is removed. Moreover, there were remained with keep the value of gray scale disappeared by the conventional CNN. Furthermore, from simulation results of edge-preserving smoothing, we have confirmed that image denoising and smoothing could be performed effectively. Moreover, from simulation results of divide domains, we could see that the thick domain and the sparse domain could be divided.

From some simulation results, we consider that the FF-CNN is effective for strengthen similar domain and not worry about small difference. In the future works, we would like to investigate the phenomena about edgepreserving smoothing by the FF-CNN.

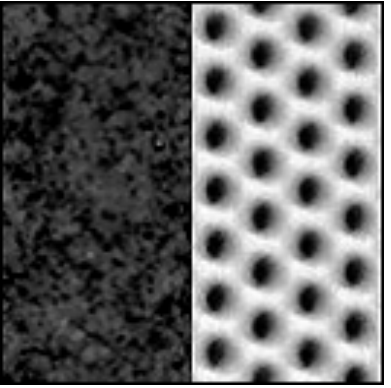

(a)

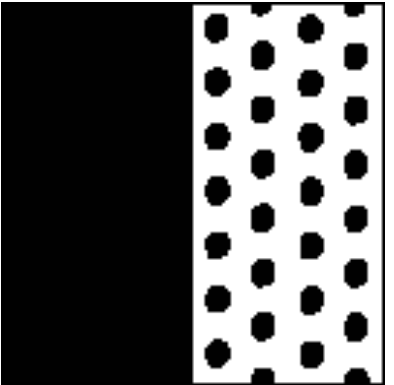

(b)
Figure 6: Simulation result by the conventional CNN. (a) Input and initial state images. (b) Output image by the conventional CNN using "S moothing" template.

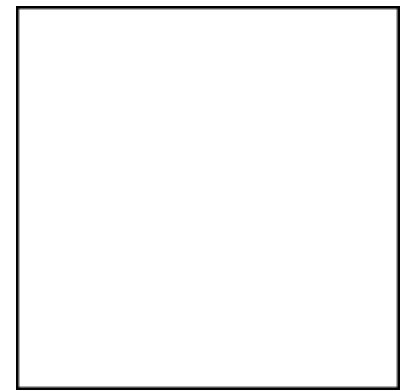

(a)

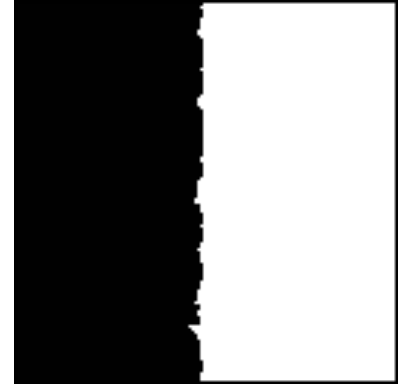

(b)
Figure 7: Divides sparse domain from thick domain result by the FF-CNN. (a) Initial state image. (b) Divide domains result by the FF-CNN using "S moothing" template.

\section{Acknowledgments}

This work was partly supported by JSPS Grant-in-Aid for Young Scientists 23700269.

\section{References}

[1] L. O. Chua and L. Yang, "Cellular Neural Networks: Theory," IEEE Trans. Circuits Syst., vol. 32, pp. 1257-1272, Oct. 1988.

[2] F. Dirk and T. Ronald, "Coding of Binary Image Data using Cellular Neural Networks and Iterative Annealing," Proc. of ECCTD'03, vol. 1, pp. 229232, Sep. 2003.

[3] M. Namba and Z. Zhang, "Cellular Neural Network for Associative Memory and Its Application to Braille Image Recognition," Proc. of IJCNN'06, pp. 4716-4721, Jul. 2006.

[4] H. Koeppl and L. O. Chua, "An Adaptive Cellular Nonlinear Network and its Application," Proc. of NOLTA'07, pp. 15-18, Sep. 2007.

[5] T. Kozek, K. R. Crounse, T. Roska and L. O. Chua, "Smart Image Scanning Algorithms for the CNN Universal Machine," Proc. of NOLTA'95, vol. 2 , pp. 707-712, 1995

[6] K. R. Crounse and L. O. Chua, "Methods for Image Processing and Pattern Formation in Cellular Neural Networks: A Tutorial," IEEE Trans. Circuits Syst., vol. 42, no. 10, pp. 583-601, Oct. 1995.

[7] Cellular Sensory Wave Computers Laboratory Computer and Automation Research Institute Hungarian Academy of Sciences, "Cellular wave Computing Library (Template, Algorithms, and Programs) Version 2.1" 